\title{
ON THE CONTRIBUTION OF OFF-NUCLEI ELECTROPRODUCTION TO THE GENERATION OF ENERGETIC HADRONS IN ELECTROMAGNETIC SHOWERS
}

\author{
H.-J. MÖHRING * \\ Deutsches Elektronen-Synchrotron DESY, Hamburg, FRG
}

Received 23 November 1989

The calculation of high-energy hadron cascades in extended materials induced by electron beams in the GeV energy range is reconsidered. In particular, we estimate the contribution from hadronic interactions of high-eriergy electrons on nuclei as compared to hadron production by photons.

Our estimate relies on the equivalent photon approximation. The interaction of the resulting quasireal photons with nuclei is described by the vector-meson dominance model and a Monte Carlo realization of the multistring fragmentation model. as discussed in ref. [1]. For thin targets (as compared to the radiation length), hadronic interactions of the incident high-energy electrons on nuclei dominate the generation of high-energy hadrons.

\section{Introduction}

So far, hadroproduction by leptons was of no importance for many practical applications in shielding and calorimetry: For example, due to the smallness of the corresponding hadronic cross sections, properties of electromagnetic calorimeters are well understood from the Monte Carlo calculation of purely electromagnetic showers in the detector geometry. However, with the construction of high-energy and/or high-current electron machines, like LEP at CERN, SLC at SLAC or CEB.AF, hadron-production processes induced by energetic electrons and photons become important, at least for shielding: The large attenuation length of hadrons is compared to leptons may compensate their small production cross sections.

For this reason, Ranft and Nelson [1] introduced a method to estimate the importance of hadron production by energetic photons in the development of electromagnetic showers. This method combines the idea of the vector-meson dominance (VMD) model with a Monte Carlo model based on the dual multistring fragmentation model. It has been included into the coupled hadron-electromagnetic cascade code FLUKA-EGS [2] for the upplication in Monte Carlo shower simulations. Note that, due to the restrictions in applicability of both the VMD and the multistring fragmentation models, the method should not be applied to photon energies below 1-2 GeV. As a consequence, reliable results are to be

* Permanent address: Sektion Physik, Karl-Marx-Universität, Leipzig, GDR. expected only for the production of hadrons with energies above about $1 \mathrm{GeV}$.

A careful comparison of calculations using the FLUKA code, with SLAC data on hadroproduction by $18 \mathrm{GeV}$ electrons on a beryllium target shows a systematic underestimation of hadron yields at high momenta (above $2 \mathrm{GeV}$ ) by a factor of order two [3]. This disagreement rises the question for other important hadron-generating mechanisms in electron-photon showers. At least for high-energy electron beams direct electron-induced hadron production on nuclei might be such a process: Although suppressed by a factor of order $\alpha / \pi$ as compared to photoproduction mechanisms, the latter ones represent two-step proresses for electron beams - the energetic photons have to be generated via hard bremsstrahlung first.

In this note we estimate the potential contribution of electron-nucleus scattering to the production of energetic hadrons within electromagnetic showers. To this end we propose an extension of the method introduced in ref. [1] on the basis of the equivalent photon approximation [4], which has been included into a working version of the FLUKA Monte Carlo code. In the following two sections we briefly describe the method and discuss some results, respectively.

\section{Extension of the photoproduction model in the FLUKA} code to hadron production by electronuclear processes

Due to the structure of the photon propagator, electron-nucleus scattering mostly takes place at low $\left|q^{2}\right|$ values of the exchanged virtual photon, with $q^{2}=\left(p_{\mathrm{e}}-\right.$ 
$\left.p_{e}^{\prime}\right)^{2}$ defined as the four-momentum transfer from the electron to the nucleus. As a consequence, the dominant fart of the electroproduction cross section may be described within the equivalent photon approximation (EPA) [4]. The essence of the EPA consists of:

1. neglecting the longitudinal degrees of freedom for the exchanged virtual photon;

2. approximating the cross section of slightly virtual transversely polarised photons by that of real ones with the same energy, $\sigma_{\mathrm{T}}\left(q^{2}\right) \simeq \sigma_{\gamma}\left(q^{2}=0\right)$.

As a result of this approximations the differential cross section may be written as [4]:

$\mathrm{d}^{\mathrm{eA}}\left(E_{\mathrm{e}}\right) \simeq \frac{N\left(x, q^{2}\right)}{x} \sigma^{\gamma \mathrm{A}}\left(x E_{\mathrm{e}}\right) \mathrm{d} x \mathrm{~d} q^{2}$,

where $x=E_{\mathrm{y}} / E_{\mathrm{e}}$ is the energy fraction of the incident electron which is transferred to the nucleus by the virtual photon. As discussed in ref. [4], the EPA is applicable up to momentum transfers $\left|q^{2}\right|=\Lambda_{\gamma}^{2}$, where $\Lambda_{\gamma}$ denotes some process-dependent dynamical cutoff; for electroproduction $\Lambda_{\gamma} \simeq m_{\mathrm{p}}$. Integrating eq. (1) over $q^{2}$ (from the lower kinematical limit $-q^{2}=m_{\mathrm{e}}^{2} x^{2} /(1-$ $x$ ) up to $\Lambda_{\gamma}^{2}=m_{\rho}^{2}$ ), results in the general, factorized form

$\operatorname{do}^{\mathrm{eA}}\left(E_{\mathrm{e}}\right)=\sigma^{\gamma \mathrm{A}}\left(x E_{\mathrm{e}}\right) \mathrm{d} n(x)$.

Since we wil! rely on the VMD approach later on, we use the "high-frequency" approximation of the photon spectrum, valid for $E_{\gamma}=x E_{\mathrm{e}} \geq \Lambda_{\gamma}=m_{\mathrm{\rho}}$ [4]:

$$
\begin{aligned}
\mathrm{d} n(x)= & \frac{\alpha}{\pi}\left[\left(1-x+x^{2} / 2\right) \ln \left[\frac{m_{\rho}^{2}(1-x)}{m_{\mathrm{e}}^{2} x^{2}}\right]\right. \\
& -(1-x)] \frac{\mathrm{d} x}{x} .
\end{aligned}
$$

This approximation describes the photon spectrum with an accuracy of

$\eta \sim\left[\ln \frac{\Lambda_{\gamma}^{2}}{-q_{\min }^{2}}\right]^{-1} \sim\left[\ln \frac{m_{\mathrm{\rho}}^{2}(1-x)}{m_{\mathrm{e}}^{2} x^{2}}\right]^{-1}$,

which is better than $5 \%$ for the dominant kinematical region at relatively small $x$ values.

With these formulae we are able to approximate the total cross section for the generation of energetic hadrons in electron-nucleus scattering by integrating eq. (2) over the allowed $x$ range $m_{\mathrm{\rho}} / \bar{E}_{\mathrm{e}} \leq x<\mathrm{1}$ :

$$
\begin{aligned}
\sigma^{\mathrm{eA}}\left(E_{\mathrm{e}}\right)= & \frac{\alpha}{\pi} \int_{m_{\mathrm{\rho}} / E_{\mathrm{e}}}^{1-\varepsilon} \sigma^{\gamma \mathrm{A}}\left(x E_{\mathrm{e}}\right) \\
& \times\left[\left(1-x+x^{2} / 2\right) \ln \left[\frac{m_{\mathrm{\rho}}^{2}(1-x)}{m_{\mathrm{e}}^{2} x^{2}}\right]\right. \\
& -(1-x)] \frac{\mathrm{d} x}{x} .
\end{aligned}
$$

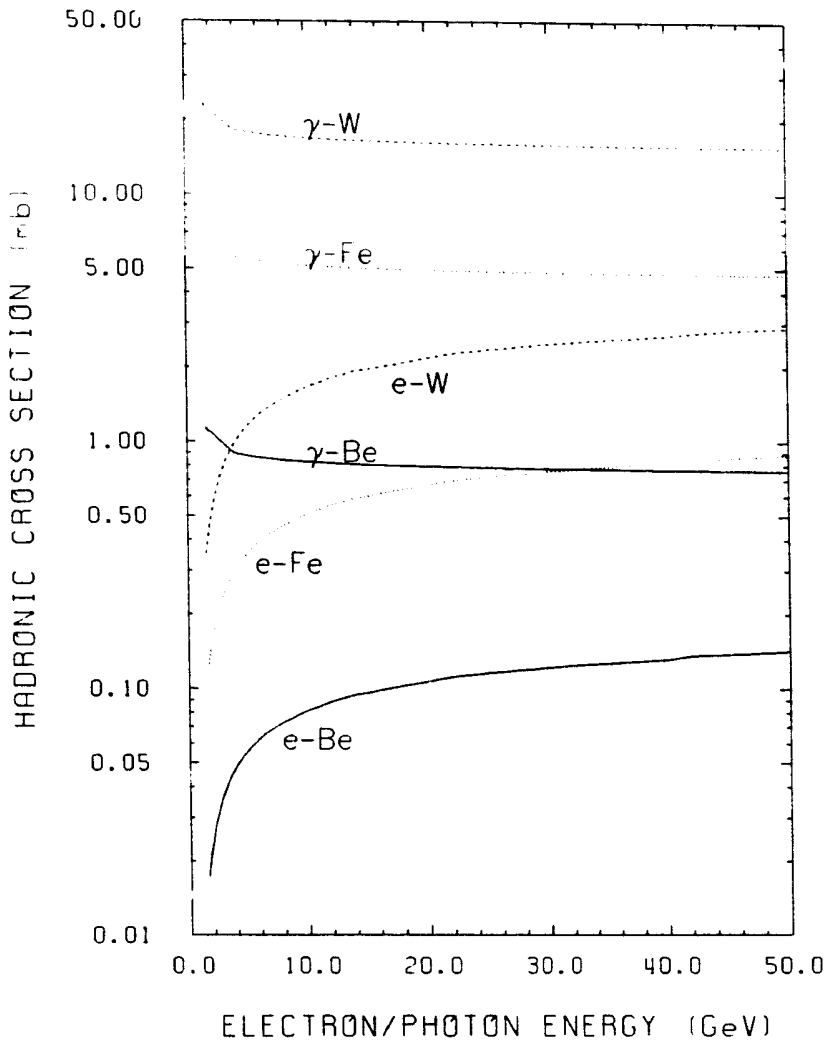

Fig. 1. Estimates for hadronic cross sections of high-energy electrons, calculated from eq. (5) for three different materials. For comparison the corresponding hadronic cross sections of photons are also shown.

(Note that the estimates within the EPA require the final-state electron to be relativistic; the method is not applicable in the limiting case $x=1$. For our actual estimates we use $\epsilon=10^{-3}$.) Results for $\sigma^{\mathrm{eA}}\left(E_{\mathrm{e}}\right)$ according to eq. (5) are shown in fig. 1 for three typical materials; the hadronic cross sections for photons (taken from ref. [5]) are depicted for comparison. Let us stress once more that eq. (5) estimates that part of the cross section for hadron production by high-energy electrons off-nuclei which may be approximated within the VMD model, and thus may result in the generation of high-energy hadrons $(E \geq \sim 1 \mathrm{GeV})$.

Based on these approximations we have extended the model of ref. [i] to the case of haủion productiotion in electron-nucleus interactions by the following procedure:

1. estima $e$ the cross section (and the corresponding mean free path) from the integral of eq. (5);

2. sample the energy ( $x$ value) of the actual "equivalent" (real) photon according to the distribution of eq. (2);

3. generate hadrons according the photoproduction model [1] already implemented in the FLUKA code. 

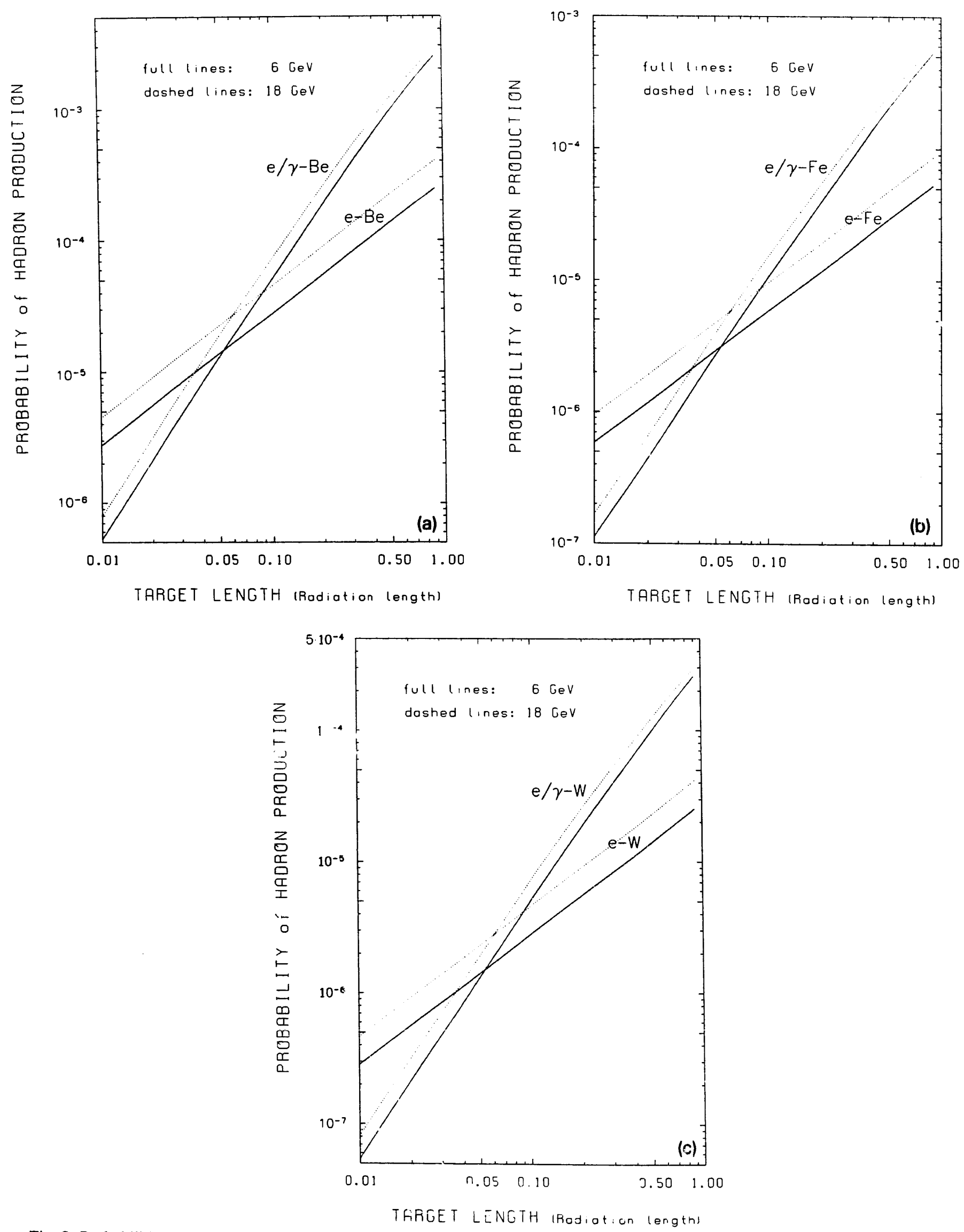

Fig. 2. Probabilities for hadron production in direct electron-nucleus scattering, $W_{\text {had }}^{\text {eAd }}$, from eq. ( 7 ), and in photonuclear reactions via real-photon bremsstrahlung, $W_{\text {had }}^{\text {e/ }}{ }^{-A}$, from eq. (6), respectively, as iunction of the target thickness $L$ (given in radiation lengths).

(a) beryllium target, (b) iron target, (c) tungsten target. 


\section{Discussion of the contribution of electronuclear reac-} tions to hadron production in electron-induced showers

To estimate the importance of direct electronuclear reactions we compare the probabilities for hadron generation in electroproduction and photoproduction processes, respectively, for an electron beam incident on a target of length $L$. For photoproduction of hadrons as a two-step process this probability can be approximated by:

$$
\begin{aligned}
& W_{\text {had }}^{\text {e/ } / \mathrm{A}}\left(E_{\mathrm{e}}, L\right) \\
& \simeq \int_{m_{\mathrm{p}}}^{E_{\mathrm{c}}} \mathrm{d} E_{\gamma} \frac{1}{\sigma_{\mathrm{brems}}^{\mathrm{eA}}} \frac{\mathrm{d} \sigma_{\mathrm{brems}}^{\mathrm{eA}}}{\mathrm{d} E_{\gamma}}\left(E_{\mathrm{e}}\right) \\
& \times \int_{0}^{L} \mathrm{~d} z \frac{1}{\lambda_{\text {brems }}^{\text {eA }}\left(E_{\mathrm{e}}\right)} \exp \left(-\frac{z}{\lambda_{\text {brems }}^{\text {eA }}}\right) \\
& \times \int_{z}^{L} \mathrm{~d} y \frac{1}{\lambda_{\text {had }}^{\gamma \mathrm{A}}\left(E_{\gamma}\right)} \exp \left(-\frac{y-z}{\lambda_{\text {had }}^{\gamma \mathrm{A}}}\right),
\end{aligned}
$$

with $\sigma_{\text {brems }}^{\text {eA }}$ denoting the cross section for real-photon bremsstrahlung from electrons scattered on atoms (taken from ref. [6]). Note that we impose the same restriction $E_{\gamma} \geq \Lambda_{\gamma} \simeq m_{\mathrm{p}}$ onto the energy of real hard protons from bremsstrahlung processes as has been applied for the estimation of $\sigma_{\text {had }}^{\text {eA }}$ in eq. (5).

This is a reasonable approximation if the target thickness $L$ is smaller than the corresponding interacting lengths for hard-photon bremsstrahlung and photohadron production, $\lambda_{\text {brems }}^{\text {eA }}$ and $\lambda_{\text {had }}^{\gamma A}$, respectively. Because of the small hadronic cross section of photons this condition is generally fulfilled for $\lambda_{\text {had }}^{\gamma \mathrm{A}}$; with growing target length, eq. (5) will underestimate the probability of hadron production due to the increasing importance of multiple hard bremsstrahlung. (The interaction length for bremsstrahlung of hard phoions $\left(E_{\gamma} \geq m_{\rho}\right)$ was calculated via the integral of the approximate differential cross section given in the EGS4 manual [6].)

This is to be compared with the probability for hadron production in direct electronuclear processes:

$W_{\text {had }}^{\mathrm{e}-\mathrm{A}}\left(E_{\mathrm{e}}, L\right)=1-\exp \left[-\frac{L}{\lambda_{\text {had }}^{\mathrm{eA}}\left(E_{\mathrm{e}}\right)}\right]$.

According to the discussion in the previous section the hadronic interaction length of electrons $\lambda_{\text {had }}^{\text {eA }}$ is obtained from the cross section of eq. (2), restricted to the interaction via hard photons $\left(E_{\gamma} \geq m_{\rho}\right)$ where the VMD model should become applicable.

Both probabilities are depicted as function of the target thickness in figs. $2 \mathrm{a}-\mathrm{c}$ for $\mathrm{Be}, \mathrm{Fe}$ and $\mathrm{W}$ targets. (Note that the target thickness is given in units of the radiation length.) Curves are shown for the energy foreseen in the CEBAF project (high-current $6 \mathrm{GeV}$ electron beam) as well as for $18 \mathrm{GeV}$ electrons for which there are SLAC data on hadron production avail-

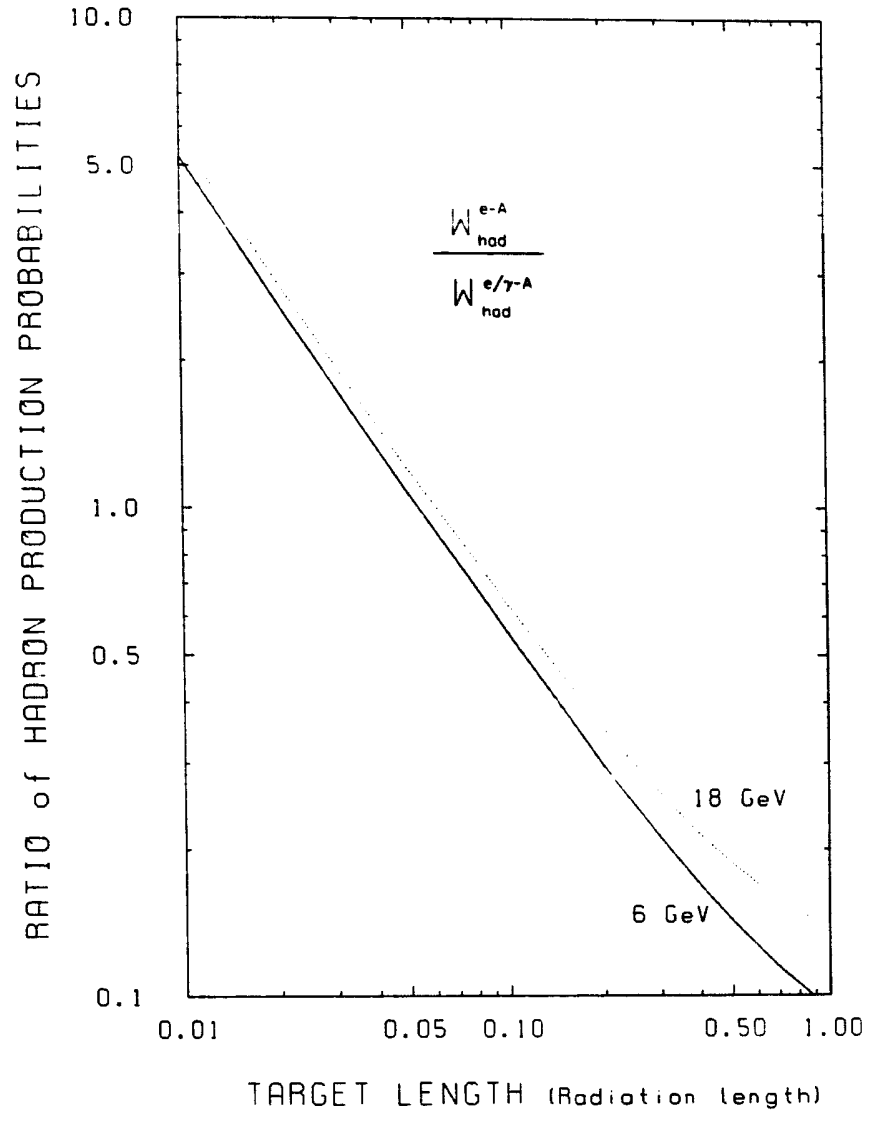

Fig. 3. Ratio of hadron-production probabilities $W_{\text {had }}^{\text {e-A }}$ $W_{\text {had }}^{\text {e/ }}-$ i vs the target thickness $L$, which is practically material-independent if $L$ is represented in radiation lengths.

able [7]. From these figures we observe that for very thin targets the production of energetic hadrons is dominated by the direct electronuclear process, but due to the large cross section for real-photon bremsstrahlung the twu-step process takes over rather soon. If the target length is scaled by the radiation length, the ratio of both probabilities turns out to be practically material-independent; it is depicted in fig. 3 for the same two electron energies as used in fig. 2.

As a consequence, direct electroproduction of hadrons cannot be expected to improve the agreement of shower calculations by the FLUKA-EGS code with the SLAC data on hadron production ( $E_{\text {had }} \geq 2 \mathrm{GeV}$ ) by $18 \mathrm{GeV}$ electrons: The corresponding experiment [7] did use a beryllium target with a length of $0.3 \lambda_{\text {rad }}$. According to our estimates, under these condictions the contribution of the direct electronuclear process does not exceed $25 \%$ of the photoproduction cross section (cf. fig. 3). Actual calculations with a modified version of the FLUKA-EGS code even give a suppression factor of about five to six to be attributed to multiple hard bremsstrahlung processes.

Summarizing our discussion we found that direct electronuclear processes will dominate the production 
of energetic hadrons (i.e. with energies above $1 \mathrm{GeV}$ ) by electron beams in targets which are thin compared to the radiation length. In such circumstances the proposed extension of the photoproduction model of ref. [1] may be useful for estimating fluxes of energetic hadrons. However, this mechanism is not expected to resolve the discussed disagreement between model calculations and the SLAC data for hadron production by $18 \mathrm{GeV}$ electrons.

\section{Acknowledgements}

The author acknowledges discussions with A. Fasso and M. Hoefert, which stimulated this work. Further helpful comments were due to W.R. Nelson, and the extension of the FLUKA code was done in contact with P. Aarnio.

\section{References}

[1] J. Ranft and W.R. Nelson, Nucl. Instr. and Meth. A257 (1987) 177

[2] P. Aarnio, J. Lindgren, J. Ranft, A. Fasso and G. Stevenson, Enhancements to the FLUKA86 Program (FLUKA87), CERN Divisional Report, TIS-RP/190 (January 1987); P. Aarnio, A. Fasso, H.-J. Möhring, J. Ranft and G. Stevenson, FLUKA86 User's Guide, CERN Divisional Report, TIS-RP/168 (January 1986).

[3] W.R. Nelson and A. Fasso, private communication.

[4] A discussion of the equivalent photon approximation, useful for our application, can be found in: V.M. Budnev, I.F. Ginzburg, G.V. Meledin and V.G. Serbo, Phys. Rep. 15 (1975) 181.

[5] T.H. Bauer, R.D. Spital, D.R. Yennie and F.M. Pipkin, Rev. Mod. Phys. 50 (1978) 261.

[6] W.R. Nelson, H. Hirayama and D.W.O. Rogers, The EGS4 Code System, SLAC-Report-265 (December 1985).

[7] A.M. Boyarski, F. Bulos, W. Busra, R. Diebold, S.D. Ecklund, G.E. Fischer, J.R. Rees and B. Richter, Yields of Secondary Particles from $18 \mathrm{GeV}$ Electrons, in: SLAC Users Handbook (1971);

ibid., Phys. Rev. Lett. 18 (1967) 363. 